Pensamiento Crítico Vol. 18 N² 2, pp. 19-41

\title{
Construyendo nuestra identidad nacional \\ La trampa del ingreso medio y la procrastinación de las reformas
}

Jorge Barrera Herrera

\section{RESUMEN}

En este ensayo, se analizan cuáles serían los principales limitantes de nuestro desarrollo institucional nacional.

Inicialmente se describe la situación económica mundial y nacional, como un preámbulo para entender el actual contexto socio económico global en el que estamos inmersos y las perspectivas a enfrentar, destacando la posible iniciación de un ciclo económico decreciente global y el término de las políticas monetarias no convencionales con tasas de interés bajas cercanas a cero, de los países avanzados.

Se estima, que el Perú, continuará siendo el país con mayor crecimiento y menor inflación de la región, sin deuda y con altas reservas internacionales, lo que nos permitirá enfrentar adecuadamente cualquier contingencia económica externa global negativa.

En lo referente a los factores limitantes de nuestro desarrollo institucional nacional, se revisan cada uno de ellos, identificando como los más importantes: terminar de construir nuestra identidad nacional, mejorar la calidad educativa, el preocupante 


\section{Pensamiento Crítico Vol. 18. № 2}

sesgo al estatu quo, la complacencia por la trampa del ingreso medio y el juego y cálculo político.

La Institucionalidad nacional, ahora más que antes, se ve comprometida por las cada vez más evidentes interconexiones de las políticas globales y los efectos de contagio, demandando nuevas y mejores acciones de coordinación entre países, todavía no alcanzadas.

Se concluye que son los valores, la afirmación de la identidad nacional, el mejoramiento de la calidad educativa, entre los más importantes factores limitantes a superar, lo que nos permitirá alcanzar la masa crítica poblacional con el nivel institucional requerido, lo que permitirá alcanzar un eficiente gestión del bien común, implementando políticas públicas sostenidas en beneficio de las grandes mayorías en una relación amigable con su naturaleza.

Palabras claves: Institucionalidad, procrastinación, diversificación cuantitativa, convergencia económica global, divergencia de ingresos, desbalances globales, valor de cambio, valor de uso.

\section{ABSTRACT}

In this paper, we analyze what would be the main limiting our national institution.

Initially described the global and national economic situation as a prelude to understanding the current global economic and social context in which we are immersed and outlook to face, highlighting the possible initiation of a global downward economic cycle the term of monetary policies conventional low interest rates close to zero, in the advanced countries.

It is estimated that Peru will continue to be the country with higher growth and lower inflation in the region, no debt and high international reserves, enabling us to adequately address any overall negative external economic contingency.

With regard to the factors limiting our national institutional development, are reviewed every one of them, identified as most important: finish building our national identity, improve educational quality, the worrying status quo bias, complacency by the trap average income and the game and political calculation.

The National Institutions, now more than ever, is compromised by the increasingly evident interconnections of global policies and spillovers, demanding new and better coordinated actions among countries not yet reached.

One concludes that the values, the affirmation of national identity, improving the quality of education, the most important limiting factors to overcome, allowing us 


\section{Jorge Barrera Herrera}

to reach critical mass population with the required institutional level, which will achieve a efficient management of public resources, implementing sustained public policies for the benefit of the majority in a friendly relationship with nature.

Keywords: Institutions, procrastination, quantitative diversification, global economic convergence, divergence of income, global imbalances, exchange value, use value.

\section{GENERALIDADES SOBRE LA SITUACIÓN ECONÓMICA MUNDIAL}

Durante largo tiempo, la economía mundial se ha estado desarrollando en condiciones excepcionalmente favorables, ubicándose en el ciclo expansivo, (ver CUADRO $\mathrm{N}^{\circ} 1$ ) situación que ha permitido aprovechar a los países emergentes y por tanto al Perú, mejorar las condiciones de convergencia económica entre los países ricos y pobres aumentando el crecimiento mundial en general (ver CUADRO $\mathrm{N}^{\circ} 2$ ).

La crisis financiera del 2008 iniciada en los Estados Unidos, que se expandió a todo el mundo y que todavía no se logra superar, puso al descubierto serios problemas de desbalances, desequilibrios y transparencia de información globales, que a pesar de los coordinados esfuerzos realizados por el Grupo de los veinte (G-20), del Fondo Monetario Internacional (FMI), Banco Mundial (BM), Bancos Centrales (BC), etc., todavía no han sido totalmente normalizados.

CUADRO $N^{\circ} 1$. Crecimiento mundial: En agosto, el porcentaje de inversionistas que espera una recuperación de la economía mundial subió de 52 a 72\% (Encuesta Global de Administradores de Fondos, GFMS).

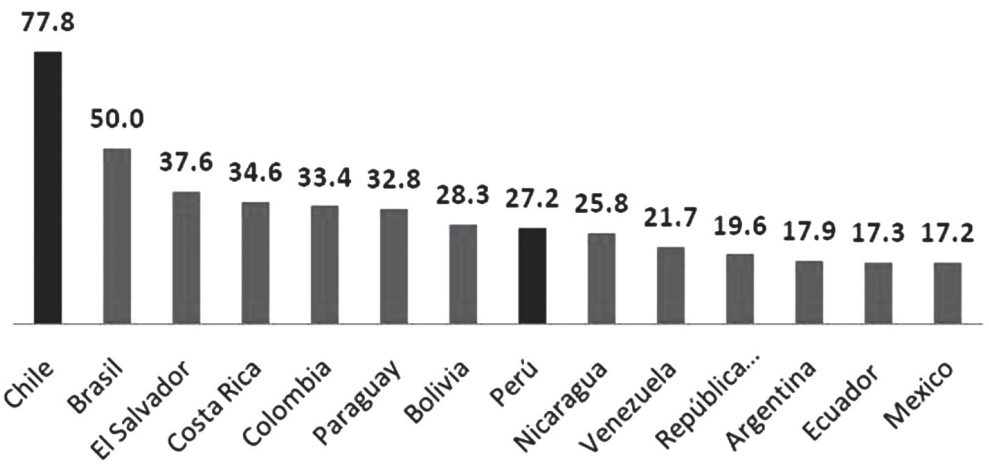




\section{Pensamiento Crítico Vol. 18. No 2}

CUADRO No 2 . Se espera una recuperación de la economía mundial a partir del segundo semestre de 2013.

\begin{tabular}{|l|c|c|c|}
\hline \multicolumn{4}{|c|}{ FMI: Proyección de crecimiento mundial } \\
\hline & $\mathbf{2 0 1 2}$ & $\mathbf{2 0 1 3}$ & $\mathbf{2 0 1 4}$ \\
\hline Mundo & 3.1 & 3.1 & 3.8 \\
\hline Desarrolladas & 1.2 & 1.2 & 2.1 \\
\hline Emergentes & 4.9 & 5.0 & 5.4 \\
\hline
\end{tabular}

A julio 2013

Como consecuencia de esta crisis, el Banco Central de los Estados Unidos (FED), se ha enfrascado en una política monetaria expansionista, no convencional, sin precedentes (diversificación cuantitativa Q-3), manteniendo las tasas de interés bajas cercanas a cero, alentando el endeudamiento, el crédito y la especulación en la economía no real, con el objetivo inicial de salvar a sus Bancos y sistema financiero, y posteriormente para reforzar su demanda a fin de evitar la recesión y disminuir su desempleo. Dos terceras partes de esta emisión de dinero ha sido colocada fuera del ámbito geográfico de los Estados Unidos motivo por el cual no han sido afectados por la inflación y más bien exportaron parte de sus problemas al mundo depreciando su moneda.

De acuerdo a la opinión de muchos analistas, mientras no se solucionen, los grandes desbalances y desequilibrios de los países desarrollados, específicamente relacionados con el sobre endeudamiento en los Estados Unidos, Europa (ver cuadro $\mathrm{N}^{\circ} 3$ ) y Japón, y la relación China, países árabes y emergentes, como financiadores de esos déficits, la crisis no habrá terminado; además el modesto $2 \%$ de crecimiento económico del PBI que alcanzaría los Estados Unidos para el 2013 no nos permite asegurar que lo peor ya pasó (ver CUADRO $\mathrm{N}^{\circ} 4$ ) ; sin embargo este país ha anunciado, el retiro en el corto plazo de la actual política monetaria expansiva, para luego revertir las condiciones de crecimiento de la liquidez mundial, para frenar la especulación financiera y alentar actividades de producción del sector real, parando la creación de burbujas y converger las grandes desigualdades entre ricos y pobres, este anuncio aunado con la desaceleración del crecimiento económico Chino, la caída de los precios de los minerales (ver cuadro $\mathrm{N}^{\circ} 5$ ), está avisando un cambio de la tendencia, ahora a la baja, del crecimiento de la económica mundial mostrando una nueva manifestación internacional de la crisis que avizoran mayores exigencias de políticas de austeridad para los países endeudados. 


\section{Jorge Barrera Herrera}

CUADRO $N^{\circ} 3$. En el segundo trimestre el PBI de la Eurozona creció 1,1\% (trimestral anualizado) luego de 6 trimestres de caída.

Eurozona: Índices de actividad PMI del JPMorgan

\begin{tabular}{lcccc}
\hline & Dic-12 & Jun-13 & Jul-13 & Ago-13 \\
\hline Servicios & & & & \\
\hline Eurozona & $\underline{47,8}$ & $\underline{48,3}$ & $\underline{49,8}$ & $\underline{51,0}$ \\
Alemania & 52,0 & 50,4 & 51,3 & 52,4 \\
Francia & 45,2 & 47,2 & 48,6 & 47,7 \\
Italia & 45,6 & 45,8 & 48,7 & n.d. \\
España & 44,3 & 47,8 & 48,5 & n.d. \\
\hline Manufactura & & & & \\
Eurozona & 46,1 & $\underline{48,8}$ & $\underline{50,3}$ & $\underline{51,3}$ \\
Alemania & 46,0 & 48,6 & 50,7 & 52,0 \\
Francia & 44,6 & 48,4 & 49,7 & 49,7 \\
Italia & 46,7 & 49,1 & 50,4 & n.d. \\
España & 44,6 & 50,0 & 49,8 & n.d. \\
\hline
\end{tabular}

Fuente: Bloomberg.

CUADRO $\mathrm{N}^{\circ}$ 4. Se mantiene recuperación gradual de mercados inmobiliario y laboral en Estados Unidos

Indicadores Macroeconómicos de Estados Unidos

\begin{tabular}{|l|ccc|}
\hline & Dic. 12 & Jun. 13 & Jul.13 \\
\hline $\begin{array}{l}\text { Tasa de desempleo } \\
\text { (\%) }\end{array}$ & 7,8 & 7,6 & 7,4 \\
$\begin{array}{l}\text { Inflación } \\
\text { (Var. \% anual) }\end{array}$ & 1,7 & 1,8 & 2,0 \\
$\begin{array}{l}\text { Ventas de viviendas existentes } \\
\text { (Var. \% mensual) }\end{array}$ & $-1,2$ & $-1,6$ & 6,5 \\
\hline
\end{tabular}




\section{Pensamiento Crítico Vol. 18. No 2}

CUADRO $\mathrm{N}^{\circ}$ 5. Los precios de exportación se mantendrían por debajo del pico de 2011, pero serían aún mayores al promedio de la última década.

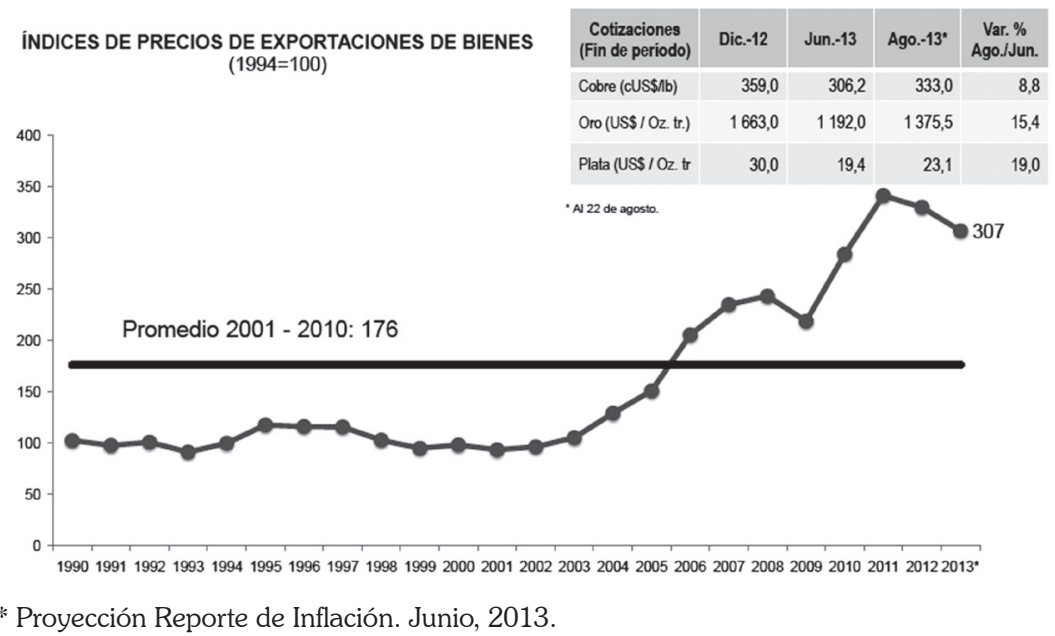

Este nuevo escenario originará, el regreso de los capitales golondrinos estacionados en búsqueda de mayores rendimientos de los países emergentes a los países desarrollados (principalmente Estados Unidos, de quien se estima subirá sus tasas de interés), ocasionando en los países emergentes presiones al alza del tipo de cambio, de la inflación y del déficit de balanza de pagos, afectando la inversión en sus mercados secundarios, además que se evidenciarán las necesarias reformas económicas postergadas, frenando el dinamismo del crecimiento económico.

Actualmente a la mayoría de países y ciudadanos del mundo, se les ha facilitado una gran deuda y la posibilidad discriminatoria de acceso a diferentes niveles de tasas de interés que los ha vuelto altamente vulnerables, expuestos, poco contestatarios y confusos, donde se observa el salvar a los bancos y el deterioro de los beneficios sociales alcanzados como una regla no democrática generalizada.

En la última reunión en Rusia del G-20 (set.2013), los países del bloque BRICS: Brasil, Rusia, India, China, Sud- áfrica; además de manifestar su defensa al uso de las redes de internet sin objetivos de espionaje, examinaron la creación de dos nuevas instituciones financieras, El Banco de Desarrollo, cuyo objetivo será proporcionar 


\section{Jorge Barrera Herrera}

los fondos necesarios para el desarrollo de proyectos de infraestructura, sin excluir la participación en el futuro de otros países; y también han mencionado la intención de crear La fundación de las Reservas de Divisas, para protegerse de los ataques contra sus monedas nacionales.

La idea en la creación de estas instituciones financieras, dada la pérdida paulatina del dólar como garantía fiable de reserva, es poder contar con alternativas nuevas a las creadas en Bretton Woods (B.M., F.M.I.-1971) que están dominadas por los Estados Unidos, de manera de poder afirmar sus monedas alejándose de la primacía del dólar, logrando disminuir los costos de transacción por sus empresas nacionales del grupo BRICS y también para ayudar al saneamiento del mercado de divisas mundial en vista del anunciado fin de la flexibilización cuantitativa en Estados Unidos.

Al igual que el despliegue, el repliegue de la política monetaria no convencional, es decir de la diversificación cuantitativa, deberá convocarnos a que juntos, países avanzados y emergentes, estemos pendientes en constatar si los beneficios de la diversificación cuantitativa generan crecimientos decrecientes y si los efectos colaterales financieros empeoran, dado que hoy todos estos resultados y efectos son desconocidos, para saber utilizar oportunamente las medidas macro y micro prudenciales, de gestión de flujo y también las líneas de canje, por parte de los Bancos Centrales, que muy buenos resultados ya han sabido dar al inicio de la crisis del 2008.

Analizar el efecto global es algo que promueve el interés propio, se deben profundizar más las interconexiones de las políticas y los efectos de contagio entre los países, es lamentable constatar que el avance de estas políticas y sus coordinaciones aún no se encuentran a la altura de las actuales exigencias que garanticen la vuelta a una sostenida normalidad de desarrollo económico y financiero global.

\section{GENERALIDADES SOBRE LA SITUACIÓN ECONÓMICA PERUANA}

El crecimiento económico peruano es impulsado con Inversión extranjera directa (IED), con alta dependencia de las exportaciones mineras, se registra un gran déficit en infraestructura especialmente en la zona no costera, es notoria la baja calidad del sistema educativo, 2/3 de la población laboral es informal reconociendo que el actual crecimiento económico no ha creado empleo estable, a pesar de haber reducido la 


\section{Pensamiento Crítico Vol. 18. No 2}

pobreza la desigualdad persiste, a pesar de que el modelo económico peruano se ha inspirado en el modelo Chileno, con la diferencia importante en nuestro país de mayores exoneraciones fiscales para la minería, China se vislumbra como el desplazante de Estados Unidos y Canadá en la IED, se ha privilegiado la estabilidad controlando la inflación y las finanzas públicas, actualmente se dispone de un fondo de estabilización fiscal para contingencias, existe fuerte dependencia de las exportaciones de recursos naturales (RRNN) y del mercado Chino mostrándose como una desventaja nuestra en el ciclo económico descendente que se prevé, la ausencia de industrialización conjuntamente con tratar de mantener el tipo de cambio bajo (apreciación moneda doméstica) ha afectado la competitividad de la industria manufacturera interna y externamente, justo a raíz del Tratado de Libre Comercio (TLC) firmado con Estados Unidos nuestro comercio con este país se ha vuelto deficitario, el riesgo país ha alcanzado grado de inversión por las principales calificadoras de riesgo internacionales.

Según datos del INEI, durante el 2012, el PBI creció 6.3\%, registrando crecimiento económico en todos los departamentos del país con excepción de Madre de Dios, explicada por la reducción de la minería ilegal. El sector construcción lideró el crecimiento en 17 departamentos encabezado por el departamento de Amazonas. Los departamentos con mayor crecimiento relativo, por encima de los S/. 20,000 nuevos soles son: Moquegua, Lima, Arequipa, Taca e Ica.

Hasta junio del 2013, la producción anual creció 5.69\%, acumulando 46 meses de resultados mensuales positivos, explicado con el crecimiento positivo de todos los sectores excepto el sector agropecuario, donde el sector comercio, servicios prestados a empresas, y construcción figuran como los más activos.

Según la SUNAT, en el 2013 las exportaciones peruanas están cayendo, los resultados de los cuatro últimos meses es preocupante, las exportaciones durante este período han alcanzado los US \$13,096 millones de dólares, un 13.3\% menor a igual período del año pasado, explicado por la menor dinámica económica de China (nuestro segundo más importante socio comercial después de Estados Unidos), por lo que la caída de los volúmenes y precios internacionales de nuestras exportaciones de materias primas y minerales han sido determinantes en la caída de nuestras exportaciones (el sector minero es el $56.2 \%$ de nuestras exportaciones). 


\section{Jorge Barrera Herrera}

Es necesario mencionar la importancia que tienen los puertos marítimos como facilitadores del transporte nacional de mercancías, como es el llamado transporte de cabotaje, en condiciones de libre mercado con supervisión estatal; debido entre otras cosas a que el $95 \%$ del transporte mundial se hace a través de los puertos marítimos contribuyendo al crecimiento económico global. En nuestro país la Ley de Reactivación y Promoción de la marina Mercante y su reglamento impiden que buques de bandera extranjera realicen cabotaje, no alentando la necesaria oferta y por ende la necesaria competencia y desarrollo de estos servicios de transporte, lo que no contribuye a disminuir los riesgos de inseguridad por robos y asaltos a los transportes (especialmente en las cercanías de los puertos), a descongestionar nuestras carreteras ya colapsadas, aumentando los sobre costos logísticos y de nuestras exportaciones, haciéndonos menos competitivos, frenando el desarrollo nacional en especial de las regiones y al interior del país. Al sector privado se le debe seguir asignando la tarea de administrar e invertir en el mejoramiento de la infraestructura de los puertos, con una adecuada negociación y supervisión del estado, dado que ha demostrado ser la mejor alternativa.

En el Perú, aunque actualmente el déficit en cuenta corriente superaría el $5 \%$ del PBI este año 2013 (ver CUADRO № 6), existen las reservas adecuadas para enfrentar la esperada desaceleración de la economía mundial (ver cuadros $\mathrm{N}^{\circ} 7$ y $\mathrm{N}^{\circ} 8$ ), también la demanda interna ha crecido en el primer semestre un $7.1 \%$, tasa superior inclusive a la del primer semestre del año pasado (ver CUADROS $\mathrm{N}^{\circ} 9, \mathrm{~N}^{\circ} 10$ y $\mathrm{N}^{\circ} 11$ ), con un incremento también de la inversión minera entre enero y mayo de $29 \%$ (ver cuadro $\mathrm{N}^{\circ}$ 12 y $\mathrm{N}^{\circ} 13$ ), y contamos con la solides de nuestros fundamentos macroeconómicos, además la posible subida del tipo de cambio permitiría contribuir a cerrar la brecha del déficit de la balanza comercial, haciendo más competitivas nuestras exportaciones, sin embargo la subida del dólar generaría también presión recesiva por las deudas contraídas en esta moneda, en ese posible escenario el Banco Central de Reserva del Perú en coordinación con el Ministerio de Economía y Finanzas, deberán establecer las medidas para controlar en forma escalonada la subida de esta divisa. 


\section{Pensamiento Crítico Vol. 18. No 2}

CUADRO $N^{\circ} 6$. Deficit de cuenta corriente financiado con entradas de capitales de largo plazo.

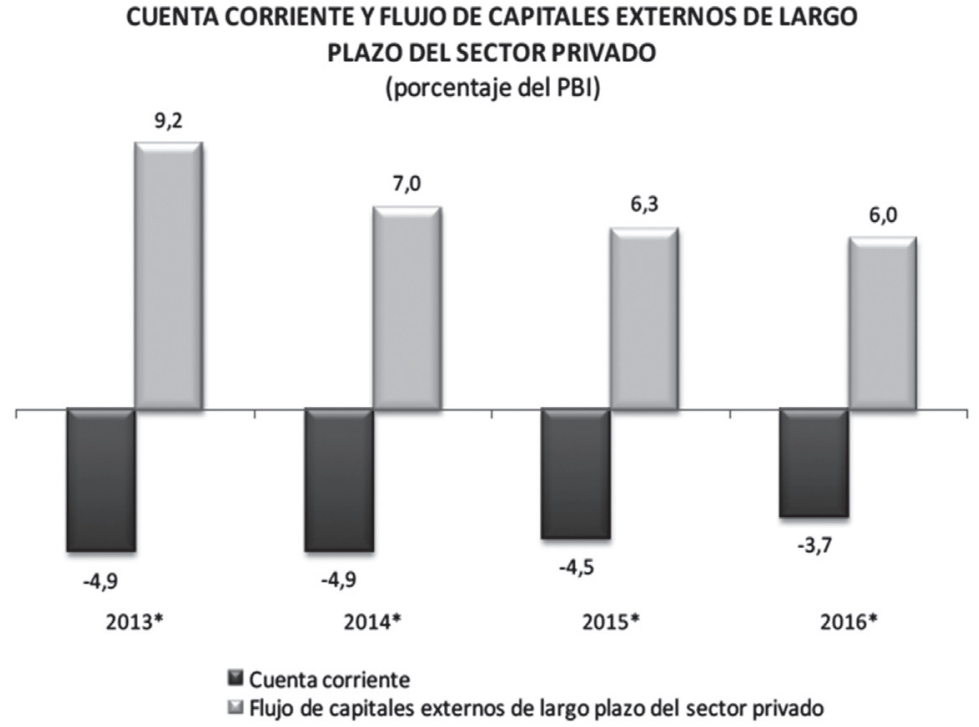

CUADRO N 7. El Banco Central continuó acumulando reservas internacionales, en un contexto de alto influjos de capitales externos hasta abril.

Reservas Internacionales Netas

(US\$ miles de millones)

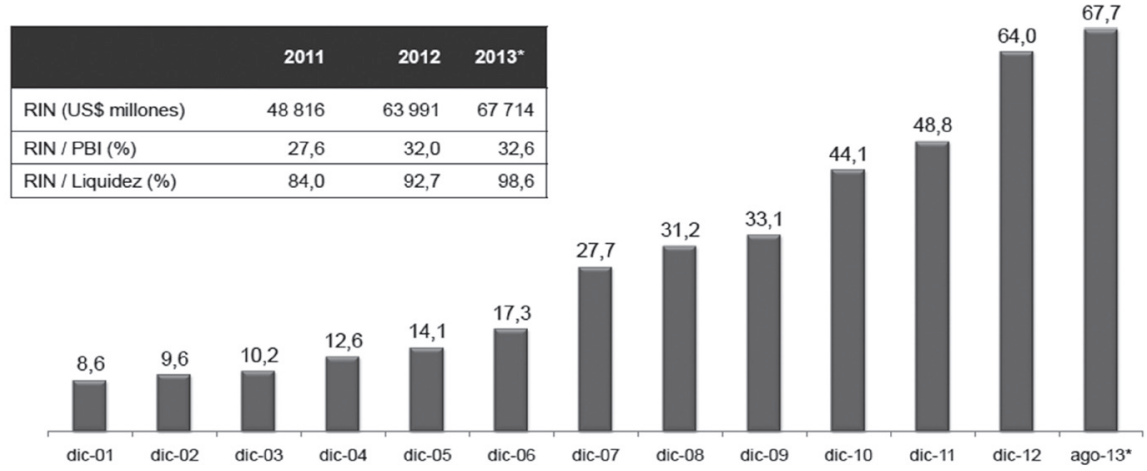




\section{Jorge Barrera Herrera}

CUADRO N ${ }^{\circ} 8$. El nivel de Reservas Internacionales del BCRP fortalece su capacidad de respuesta ante choques financieros externos, diferenciándonos de otros países.

\section{Reservas Internacionales}

(Porcentaje del PBI, 2013)

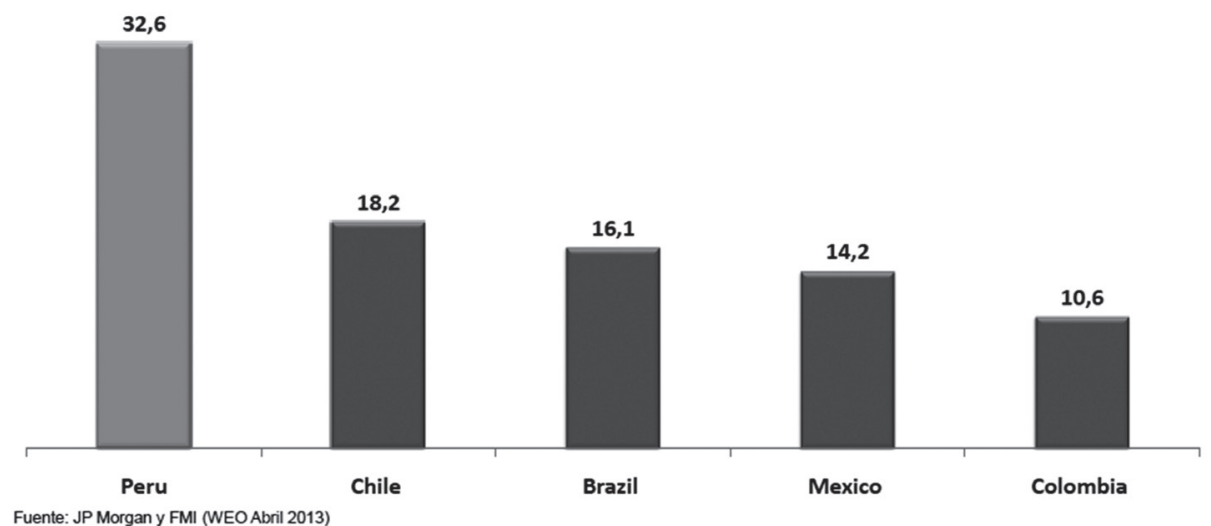

CUADRO $\mathrm{N}^{\circ}$ 9. Se mantiene el dinamismo de la demanda interna, compensando menores exportaciones netas.

\begin{tabular}{|c|c|c|c|}
\hline & \multicolumn{2}{|c|}{$\begin{array}{c}\text { Demanda Interna y PBI } \\
\text { (Variación porcentual doce meses) }\end{array}$} & \multirow[b]{2}{*}{ I Semestre 2013} \\
\hline & I Semestre 2012 & II Semestre 2012 & \\
\hline Demanda interna & 6,2 & 8,6 & 7,1 \\
\hline PBI & 6,2 & 6,3 & 5,1 \\
\hline
\end{tabular}




\section{Pensamiento Crítico Vol. 18. $\mathrm{N}^{\circ} 2$}

CUADRO $N^{\circ} 10$. Indicadores económicos muestran que se mantiene el dinamismo del mercado interno.

Producción de Electricidad: 2012 - 2013

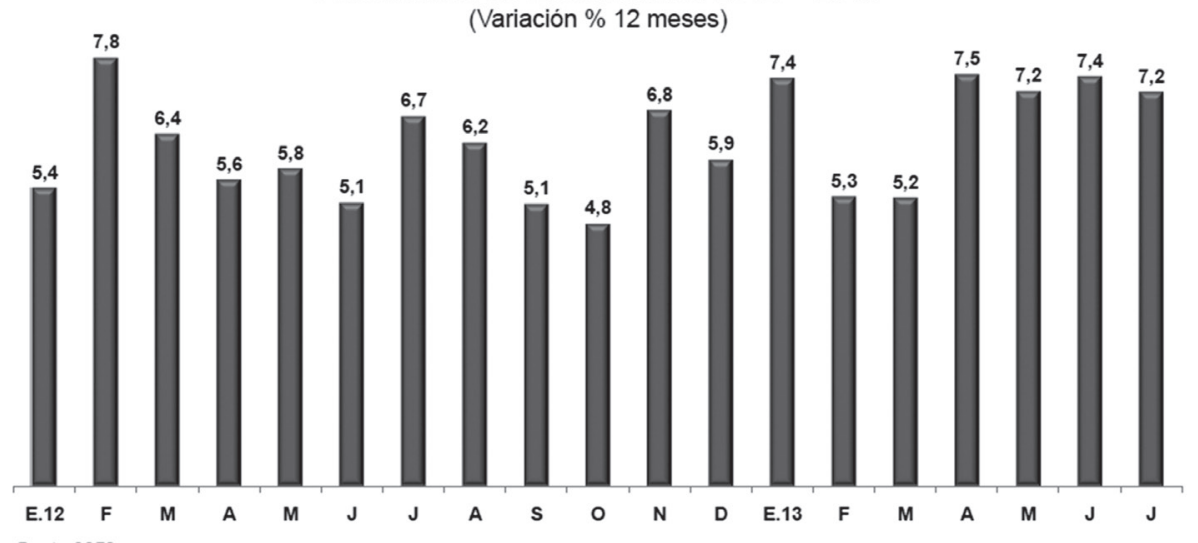

Fuente: COES

CUADRO $\mathrm{N}^{\circ}$ 11. La recuadación de IGV refleja el alto crecimiento de la demanda interna.

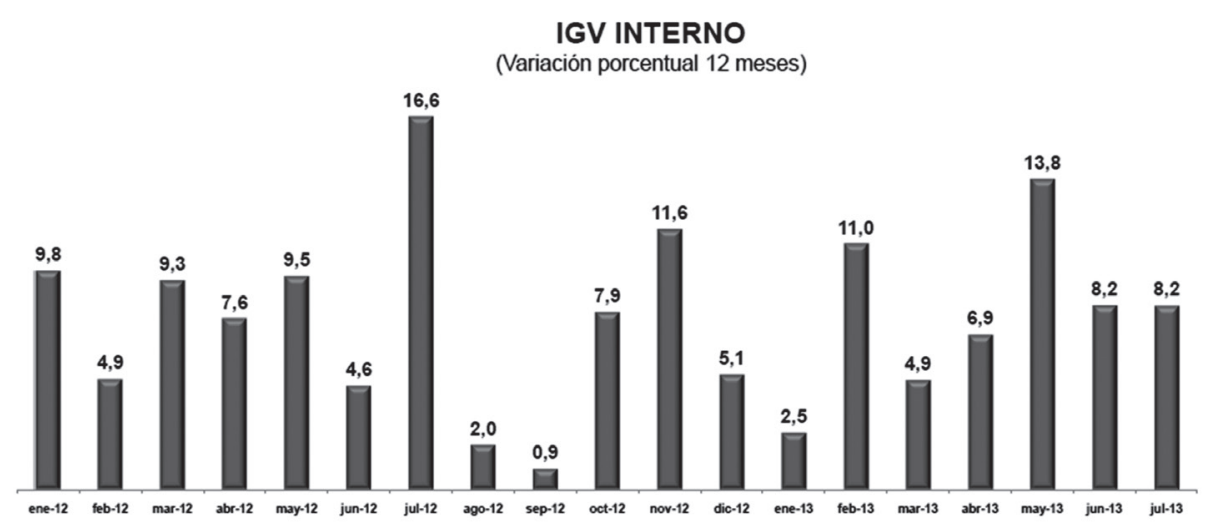




\section{Jorge Barrera Herrera}

CUADRO $\mathrm{N}^{\circ} 12$. Alto crecimiento de la inversión minera.

\begin{tabular}{|c|c|c|c|c|}
\hline \multicolumn{5}{|c|}{ INVERSIÓN MINERA } \\
\hline \multicolumn{5}{|c|}{ (millones US\$) } \\
\hline $\begin{array}{l}\text { PRINCIPAL } \\
\text { PROYECTO }\end{array}$ & EMPRESA & Ene.-May.2012 & Ene.-May.2013 & Var. \% \\
\hline Las Bambas & Xstrata Las Bambas & 332 & 727 & 119,0 \\
\hline Toromocho & Chinalco & 297 & 365 & 22,7 \\
\hline Cerro Verde & Cerro Verde & 188 & 312 & 65,9 \\
\hline Antapaccay & Xstrata Tintaya & 164 & 241 & 46,9 \\
\hline Antamina & Antamina & 262 & 186 & $-29,1$ \\
\hline Yanacocha & Yanacocha & 465 & 167 & $-64,1$ \\
\hline La Arena & La Arena & 110 & 85 & $-22,3$ \\
\hline $\begin{array}{c}\text { Ampliación Lagunas } \\
\text { Norte }\end{array}$ & Barrick Misquichilca & 58 & 83 & 43,2 \\
\hline Resto & & 1068 & 1629 & 52,5 \\
\hline TOTAL & & 2944 & 3795 & 28,9 \\
\hline
\end{tabular}

CUADRO $\mathrm{N}^{\circ} 13$. Crece la cartera de proyectos de inversión privada con respecto a diciembre del año pasado.

\begin{tabular}{l|r|r|}
\hline \multicolumn{1}{|c|}{$\begin{array}{l}\text { CARTERA PROYECTOS DE INVERSIÓN } \\
\text { PRIVADA } \\
\text { (Millones de US\$) }\end{array}$} \\
\hline & $\begin{array}{c}\text { DICIEMBRE } \\
2012\end{array}$ & $\begin{array}{c}\text { JULIO } \\
\text { (2013 }\end{array}$ \\
\hline Minería & 29568 & 34024 \\
\hline Hidrocarburos & 6896 & 9378 \\
\hline Electricidad & 5806 & 6889 \\
\hline Industrial & 2311 & 3240 \\
\hline Infraestructura & 2438 & 3413 \\
\hline Otros Sectores & 5319 & 8270 \\
\hline Total & & 65214
\end{tabular}

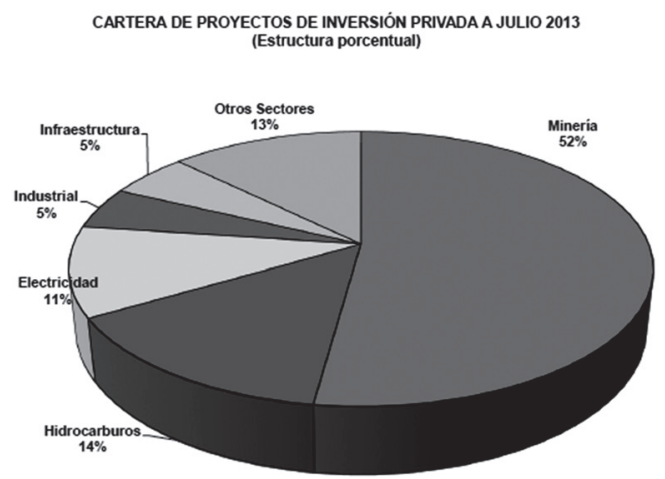

Fuente: Informes en Diarios, Medios Especializados 


\section{Pensamiento Crítico Vol. 18. No 2}

El Perú continua siendo el país con mayor crecimiento de la región y menor inflación (ver CUADRO $\mathrm{N}^{\circ} 14$ y $\mathrm{N}^{\circ} 15$ ), el fisco tiene una posición sólida (ver CUADRO $\mathrm{N}^{\circ} 16$ ) y la reciente mejora en la calificación de riesgo refleja las buenas expectativas de crecimiento y las altas reservas internacionales alcanzadas.

CUADRO № 14 . Perú continúa siendo uno de los países con mayor crecimiento.
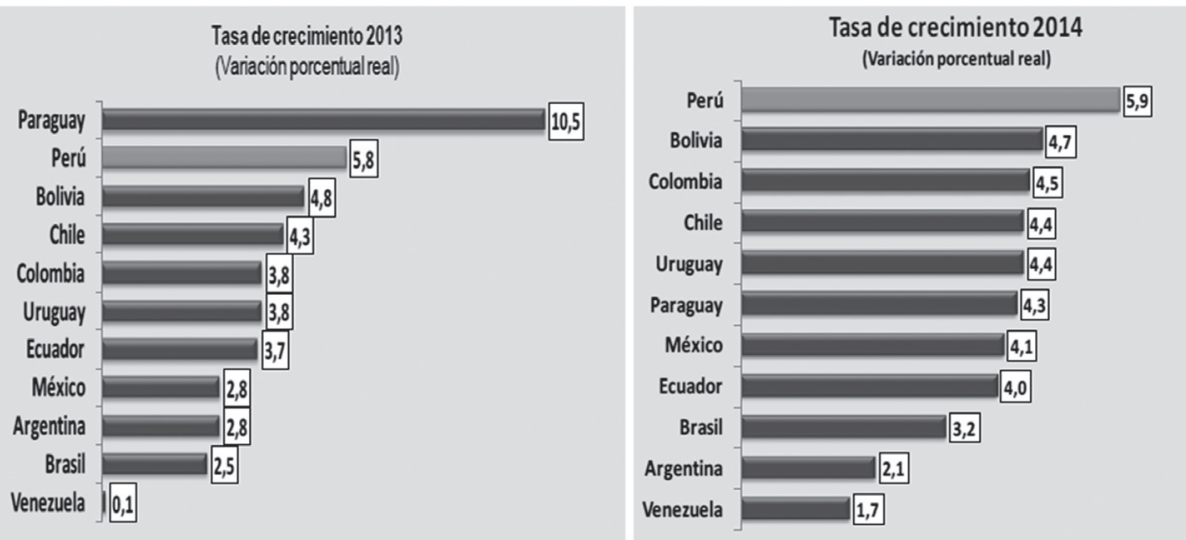

CUADRO N 15. En julio la tasa de inflación se ubicó en 3,24 por ciento.

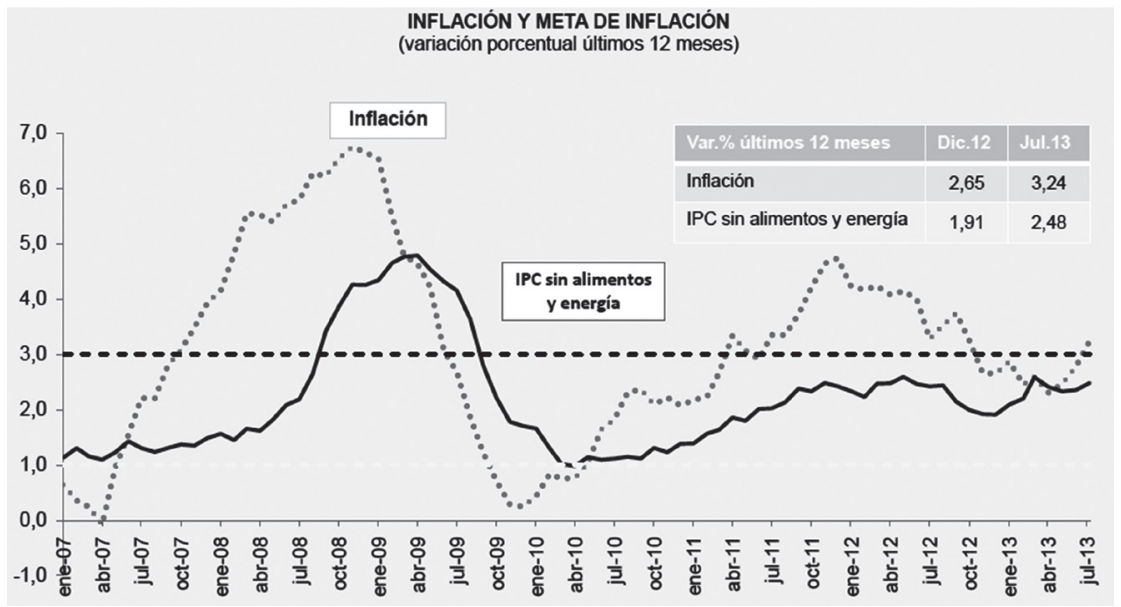




\section{Jorge Barrera Herrera}

CUADRO N 16 . Deuda Bruta y Neta del sector público no financiero (Porcentaje del PBI)

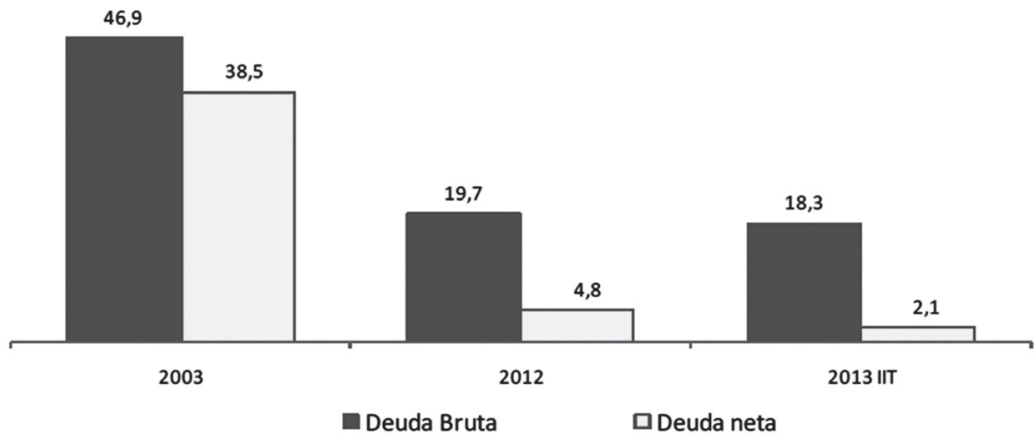

\section{CONSTRUCCIÓN DE LA INSTITUCIONALIDAD NACIONAL}

En la actualidad, los países emergentes como el Perú, requieren alcanzar una masa crítica poblacional de madurez institucional mínima para garantizar un proceso de crecimiento y desarrollo nacional sostenido, y poder hacer las reformas requeridas y aplicar políticas públicas de interés nacional con la velocidad y oportunidad, que favorezcan a las grandes mayorías.

Su construcción y vigencia de esta masa crítica poblacional de madurez institucional nacional mínima requerida, se hace más necesaria y evidente aún, por las mayores presiones, empoderamientos y demandas ciudadanas que el país comienza a adquirir, por los avances alcanzados de crecimiento económico.

¿Cuáles son los principales factores que limitan el crecimiento institucional de un País? ¿Qué hace que los gobiernos no hagan o pospongan para mañana (procrastinación) reformas estructurales de evidente necesidad nacional, con la pasividad de la creciente clase media y el desinterés de las actuales y desorganizadas fuerzas políticas?

Enumeraremos algunos de estos factores limitantes del desarrollo institucional nacional: 


\section{Pensamiento Crítico Vol. 18. No 2}

1. En el Perú, todavía la identidad nacional no ha terminado de construirse, principalmente por los problemas en la sistematización de la búsqueda de consensos en la gestión del bien común, que acarrea nuestra gran diversidad geográfica, étnica, cultural, que es también nuestro gran potencial; esto permitirá asegurar la continuidad y sostenibilidad de políticas de estado.

2. Es necesario tener una educación de calidad y acceso a la información, que permita desarrollar las capacidades requeridas para poder atender la diversidad de proyectos nacionales que demandan las grandes mayorías y el interés nacional, en el marco de la globalización.

3. Los gobiernos en vías de desarrollo, como el Perú, para hacer las necesarias reformas, deben enfrentar al siempre presente sesgo del statu quo, debido a que los grupos minoritarios perjudicados con la necesaria reforma nacional, generalmente son de mayor acceso al poder, más organizadas y oligárquicas, son las que se oponen e impiden que las reformas se lleven a cabo por los gobiernos de turno.

4. En los países, cuando se comienza a alcanzar un crecimiento económico inusual, se tiende a caer en la trampa del ingreso medio por la creciente clase media, que sumida en la complacencia y el goce del nuevo confort que da el acceso al consumo y entretenimiento, posterga enfrentar los retos de la implementación de las necesarias reformas nacionales, no exigiendo ni reclamando su realización.

5. También está, el juego y cálculo político; donde se interponen intereses de partido, mayormente de grupo, antes que los intereses nacionales, los cuales muchas veces no apoyan las necesarias reformas nacionales, dado que los beneficios políticos de la necesaria reforma se producirían en un plazo que excede el actual período de gobierno, o uso del poder, no asegurándoles el rédito político para ellos deseado.

A continuación haremos una descripción mayor sobre los factores enumerados anteriormente: 


\section{Jorge Barrera Herrera}

\section{La identidad nacional y el tratamiento del bien común:}

Es necesario tomar conciencia en la relación de equilibrio entre el hombre con la naturaleza exterior, de la importancia de la conservación de los recursos naturales renovables, no renovables y del medio ambiente, de la necesidad de industrializarnos y dar mayor valor agregado a los procesos productivos con el uso de la tecnología, y de realizar inversiones mineras, agro-industriales, etc., con la respectiva explotación de recursos naturales que esto conlleva para aumentar el consumo y las exportaciones, favoreciendo nuestro crecimiento económico en forma sostenida.

Se deberá construir una identidad nacional que nos permita lograr evitar la ruptura del necesario equilibrio de la relación de intercambio entre el hombre y su naturaleza exterior, relación socio-ambiental que además debe revisar el actual modo de acumulación del capital, tener en cuenta lo que en economía se conoce como la tragedia de los comunes, el problema del polizón, el dilema del prisionero, las fallas del mercado, las implicancias de la eficiencia de Pareto, interiorizar las externalidades en las evaluaciones de proyectos consiguiendo la licencia social y dar mayor importancia al valor de cambio en un mundo orientado por un progreso lineal en un planeta de recursos agotables que debe beneficiar a la mayoría poblacional en forma eficiente.

Si existe un intercambio de algo con el hombre, este se da con la naturaleza que lo rodea, siendo el trabajo el mediador. Es el trabajo el creador del valor de uso que combina la sustancia natural con el trabajo, lo que no sucede con el valor de cambio que es un valor social que se combina con el precio-dinero y que no contiene ninguna sustancia natural.

En el capitalismo, el valor de uso está subordinado al valor abstracto, lo preponderante es el valor de cambio, que se relaciona con la sociedad, el precio y el dinero, que puede estar completamente desconectado del valor del trabajo concreto que es el esfuerzo actualizado del trabajo real utilizado, y con la utilidad de satisfacer una necesidad humana; prima la ganancia sobre cualquier otro fin, sin consideración del perjuicio que se le puede estar causando a la naturaleza, al medio ambiente, al bien común, en último caso 


\section{Pensamiento Crítico Vol. 18. No 2}

a poder atender una necesidad humana insatisfecha que debería ser el fin de la existencia de las cosas, promoviendo el egoísmo en desmedro de la solidaridad. Esto es lo que incentiva al rompimiento del necesario equilibrio entre el intercambio del hombre y su naturaleza que aseguran su sostenibilidad y subsistencia.

Me explico, el valor de cambio, es un valor que se produce para que la mercancía pueda ser intercambiada, se formula en la producción y se sanciona en el momento de la venta o circulación, su valor dinerario o monetario es consustancial a que se concrete la venta cuya realización nunca está asegurada.

En un proceso de producción, para que no se produzcan pérdidas y se generen las ganancias requeridas, primero hay que asegurar que se produzca la venta, para lo cual es necesario que el valor del trabajo social medio incorporado en la fabricación del producto (utilización y transformación de recursos naturales externos por la acción del hombre) coincida con el valor del trabajo abstracto o social que es el que comparte el poseedor del dinero o potencial comprador.

Es posible que en el logro de mayores ganancias, esta coincidencia del valor del trabajo social medio incorporado al producto con el valor del trabajo abstracto o social este muy por encima de su valor de uso, que es el que verdaderamente responde a las necesidades y preferencias personales por satisfacer; además hay que tener en cuenta que la magnitud del valor de cambio normalmente se expresa a través del precio dentro de un sistema monetario que tampoco necesariamente responde a las demandas de las necesidades y preferencias sociales.

Dado que la ganancia es el motor de la actual organización social imperante, es a través del uso de nueva tecnología y la publicidad lo que ha permitido asegurar las ventas continuadas y también aumentar la brecha entre el valor de cambio (al que pueden acceder los poseedores del capital creando el sobre consumo), y el valor de uso al que deberían poder acceder los necesitados.

Por tanto es necesario mantener un equilibrio entre el sistema de reproducción de la naturaleza (explotación minera, agroindustrial, etc.) y el sistema de 


\section{Jorge Barrera Herrera}

creación del capital fijo (maquinarias, carreteras, puentes, etc.), para esto se deberá tomar conciencia de las implicancias del manejo del bien común, de las externalidades, de las consecuencias de los efectos ecológicos y sociales de la sobre explotación, producción y consumo; del calentamiento global y del deterioro del medio ambiente, de la importancia del agua para la vida, revisar el gran protagonismo que ahora tiene el mercado en desmedro de la solidaridad y en el fomento del egoísmo.

En resumen, se necesita construir una identidad nacional, el realizado Acuerdo nacional para luego un Proyecto Nacional va en este sentido, que reconozca todos los proyectos de la existente gran diversidad étnica, geográfica, social y cultural peruana basados en el bien común y que contemple la posibilidad de poderlos realizar, tomando en cuenta el necesario equilibrio del hombre con su naturaleza mencionado anteriormente, esta identidad nacional nos permitirá tener definidas claramente la preferencias, nos facilitará la creación de procesos o negociaciones que nos permitan alcanzar los necesarios consensos en las naturales pugnas de interés para poder continuar las políticas públicas, alcanzando mayor bienestar y felicidad para todos desalentando acciones especulativas que no crean valor real, lo cual nos hará elegir completamente libres, ser menos manipulables por los países desarrollados o intereses subalternos (no nacionales) y también poder asumir con responsabilidad y conciencia las consecuencias de nuestras decisiones.

\section{Educación de calidad}

Son los proyectos nacionales comprometidos con el crecimiento económico, los que demandan la necesidad de mayores competencias de los ciudadanos para su realización y ejecución, esto es lo que empuja y promueve el rol educativo que deben satisfacer los colegios, universidades y centros educativos nacionales en general.

En el Perú, es la educación una verdadera prioridad, dado que nos encontramos en casi todos los rankings internacionales en los últimos puestos sobre aspectos relacionados al tema enseñanza-aprendizaje. 


\section{Pensamiento Crítico Vol. 18. No 2}

El problema tiene que ver, además del histórico escaso presupuesto, también con la baja calidad de los profesores y las dificultades de poderlos capacitar o reemplazar, por las trabas a resolver sobre consideraciones de derechos laborales, de repercusiones e intereses políticos y sociales de larga data.

El proceso de enseñanza aprendizaje de los centros de estudios nacionales, primario, secundario, institutos, pre y post-grado, deben tener la capacidad de desarrollar adecuadamente las capacidades y habilidades demandadas y requeridas por el empresariado, por las industrias y por la sociedad en su conjunto; esto alentará la movilización social, el empoderamiento de la ciudadanía y por ende el crecimiento económico; en caso contrario se retrasará el proceso de concientización de las fuerzas, debilidades, amenazas y oportunidades del país en el avance de la institucionalidad postergando decisiones importantes para después.

Se deberán crear las condiciones para que la ciudadanía pueda acceder a la información nacional y global, utilizando todos los medios modernos de comunicación disponibles, regulando para que se fomente e impere la transparencia y oportunidad de la información, para su eficiente utilización en la toma de decisiones.

Los sistemas educativos nacionales, dejarán de ser tan marcadamente autoritarios y basados en el desprecio hacia el otro, deben promover en los estudiantes aprender a aprender dejando de ser repetidores de dogmas tradicionales muchas veces desactualizados y fuera de contexto, deberán promover los procesos de investigación y si es necesario importar tecnología de punta para adecuarla a nuestra realidad, diversificándonos para no ser solo primario exportadores, tratando de industrializar la producción del consumo nacional y las exportaciones dándoles un alto valor agregado y contenido local, dotando profesionales altamente calificados.

\section{La trampa del ingreso medio}

La trampa del ingreso medio, se puede considerar como un efecto colateral del crecimiento económico. Cuando una sociedad o país logra alcanzar 


\section{Jorge Barrera Herrera}

implementar un sistema de producción masivo con facilidades de acceso a nuevas tecnologías y además ha logrado a través de la publicidad, inducir y estandarizar la mayoría de las necesidades sociales, es porque esa sociedad necesariamente a alcanzado un ingreso medio que le permite no solo satisfacer plenamente sus necesidades básicas, sino también lograr tener un excedente para el sobre consumo y el entretenimiento; es en estas circunstancias que la sociedad se muestra conformista con lo que tiene, sin voluntad de hacer mayores cambios cuando siente su ciudadanía poco empoderada y fácilmente manipulada por el Estado para el continuismo de la gobernabilidad, a pesar de existir evidentes necesidades de reformas estructurales y cambios nacionales cuya no realización pueden poner en riesgo la velocidad y sostenibilidad del crecimiento económico del país.

Este aletargamiento y confort social desincentiva la movilización social que es la que impulsa al individuo a superarse y querer satisfacer necesidades superiores a la de los demás y a exigir se realicen las reformas requeridas por el estado. Es cuando se produce el frenazo o la trampa del ingreso medio.

Es el frenazo o estancamiento en el crecimiento económico, que suele ocurrir en los países en vías de desarrollo, cuando estos alcanzan un ingreso medio por encima de los S/. 20,000 nuevos soles per cápita, debido a que en países con tasas de inversión elevadas se puede prever a futuro un bajo rendimiento de capital y tipos de cambio reales subvaluados, proporcionando un desincentivo para elevar la escala tecnológica pocas veces exigida por el conformismo social alcanzado.

En países emergentes con crecimiento económico relevante, es necesario no caer en la trampa del ingreso medio y la complacencia por el estatu quo que esto conlleva, implementado políticas públicas de estado, principalmente la educación y sistema de tributos con adecuados programas sociales en su redistribución para disminuir desigualdades, que faciliten la movilidad social, de modo que a la sociedad se le asegure que con su esfuerzo y aporte en interés propio y comunitario es factible lograr una mayor superación y bienestar. 


\section{Pensamiento Crítico Vol. 18. No 2}

\section{El juego político}

En el Perú, desde ya varias décadas, desde que se cerró el congreso en 1992, la distancia de los electores y los partidos políticos es cada vez mayor, estos no responden a las necesidades y preferencias de las mayorías nacionales. Son grupos de presión desorganizados sin voluntad de hacerse una reingeniería que les permita acercarse más a su elector o militante, y tener la voluntad de establecer nombramientos de autoridades partidarias a través de procesos democráticos universales, predeterminados y transparentes; esto ha motivado que actualmente la mayoría de partidos políticos sean agrupaciones colectivas articuladas fuertemente con la persona candidato, que solo tienen vigencia y notoriedad en los procesos electorales y que no tienen un programa político claro diferenciado.

Las izquierdas y derechas nacionales se limitan a repetir fórmulas y no se detienen a reflexionar sobre sus carencias, posibilidades y limitaciones, donde prima la lógica del caudillaje y el clientelismo.

Es en este contexto, en el que el juego político de los llamados partidos, ponen en acción sus intereses de grupo para el cálculo del "toma y daca", es decir que es lo que nosotros vamos a recibir para ver qué es lo que nosotros te aprobamos o te damos como parte del poder, en el congreso, comisiones, etc., sin hacer un análisis profundo de los costos y beneficios de los proyectos; muchas veces se postergan las necesarias reformas nacionales debido a que sus resultados se darán en el largo plazo, en otro gobierno y sus beneficios no serán capitalizados por el grupo político, sin importar la mayoría de las veces los resultados en función del beneficio al país, del aprovechamiento de las oportunidades, ayudas y coyunturas nacionales y globales, en desmedro de los intereses de las mayorías nacionales, corrompiendo, frenando y enturbiando el crecimiento económico nacional, imponiéndose el interés de grupo por mantener o conseguir privilegios a costa del resto del país. También en la práctica, los grupos políticos se comportan como cualquier grupo de personas minoritarias afectadas por las consecuencias negativas de una necesaria reforma de estado en favor de las mayorías (sesgo al estatu quo), oponiéndose e impidiendo su ejecución, recurriendo inclusive, para tal efecto, a sus posibilidades de fácil acceso al poder de turno. 


\section{Jorge Barrera Herrera}

\section{CONCLUSIONES}

En definitiva, son los valores, la afirmación de la identidad nacional, lo que nos permitirá alcanzar la masa crítica poblacional con el perfeccionamiento institucional requerido, principalmente del estado que garantice una eficiente intervención en el manejo del bien común. Es necesario, que con el uso de la razón, de la conciencia nacional, de nuestras relaciones internacionales y de la información disponible; se logre desalentar acciones de reinversión especulativa que no crea valor real, evitando postergar y hacer las reformas estructurales requeridas, para conseguir finalmente disminuir el desempleo y la pobreza, que permita al trabajador individual alcanzar libremente en el mercado, una relación justa entre lo que produce con su trabajo para satisfacer una necesidad y lo que le es realmente pagado, promoviendo la movilización social requerida que permita alcanzar un sostenido mayor bienestar y seguridad social para las grandes mayorías nacionales, en un ambiente de equidad y solidaridad con los demás, buscando el equilibrio adecuado para lograr la demandada protección y conservación sobre la explotación de los recursos naturales y el medio ambiente, asegurando la sostenibilidad de la vida en nuestro actual mundo globalizado. 